\title{
Crecimiento y productividad de dos genotipos de maní (Arachis hypogaea L.) según densidad poblacional establecidos en Nuble, Chile
}

\author{
Growth and productivity in relation to population density for two peanut \\ (Arachis hypogaea L.) genotypes in Nuble Province, Chile \\ Nelson Zapata $^{1 *}$, Marisol Vargas $^{1}$, Felipe Vera $^{1}$
}

\begin{abstract}
RESUMEN
El objetivo de este trabajo fue estudiar el efecto de la densidad poblacional sobre el crecimiento y productividad de maní tipo Español y Virginia cultivados en Nuble, Chile. Con cada genotipo se estableció un ensayo de campo en diseño de bloques completos al azar con cuatro repeticiones, considerando cuatro densidades poblacionales entre 104 y 417 mil plantas ha ${ }^{-1}$. La unidad experimental $\left(12 \mathrm{~m}^{2}\right)$ contenía cuatro hileras espaciadas a $60 \mathrm{~cm}$. La cosecha se realizó 152 días después de siembra (DDS). Entre los 73 y 152 DDS se determinó área foliar, biomasa acumulada/órgano, número de órganos por planta e índice de cosecha. A cosecha se extrajeron las plantas de $3,6 \mathrm{~m}^{2}$ y se determinó peso seco de parte aérea, frutos y semillas. Para ambos genotipos el aumento de la densidad poblacional disminuyó significativamente el área foliar, número de órganos y biomasa acumulada por planta. La distribución de biomasa en la planta no resultó afectada. Por unidad de superficie, mayores densidades implicaron mayor rendimiento de semilla (2.031 y $1.932 \mathrm{~kg} \mathrm{ha}^{-1}$ para los genotipos Español y Virginia, respectivamente). Altas densidades poblacionales en maní tipo Español y Virginia disminuyen el crecimiento por planta, sin embargo, por unidad de superficie, incrementan significativamente la productividad.
\end{abstract}

Palabras clave: Arachis hypogaea L., leguminosas de grano, cultivos anuales.

\begin{abstract}
The aim of this study was to analyze the effect of population density on growth and productivity of Spanish and Virginia peanuts grown in Nuble, Chile. For each peanut genotype a field trial was established using a randomized complete block design with four replications. Four population densities between 104 and 417 thousand plants ha ${ }^{-1}$ were used. The experimental unit $\left(12 \mathrm{~m}^{2}\right)$ consisted of four rows spaced at $60 \mathrm{~cm}$. The harvest took place 152 days after sowing (DAS). Between 73 and 152 DAS the leaf area, biomass accumulated per organ, number of organs per plant and harvest index were determined. At harvest, plants contained in $3.6 \mathrm{~m}^{2}$ of each plot were removed, and dry weight of aerial parts, fruits and seeds was determined. For both peanut genotypes, increasing population density decreased leaf area, number of organs and accumulated biomass per plant. The distribution of plant biomass was unaffected. Greater density produced higher seed yield per unit area (2,031 and 1,932 $\mathrm{kg} \mathrm{ha}^{-1}$ for Spanish and Virginia genotypes, respectively). High population densities in Spanish and Virginia peanuts reduce individual plant growth, but increase productivity per unit area.
\end{abstract}

Key words: Arachis hypogaea L., legumes, annual crops.

\section{Introducción}

El maní (Arachis hypogaea L.) es una leguminosa muy valorada a nivel mundial, está presente en la dieta de gran parte de la población y para muchos pueblos constituye la principal fuente de proteínas y lípidos. El maní que se cultiva actualmente comprende las subespecies hypogaea y fastigiata y a nivel comercial se distinguen principalmente cuatro tipos; Runner y Virginia (var. hypogaea), Español (var. vulgaris) y Valencia (var. fastigiata) (Knauft et al., 1987), siendo el tipo Runner el más transado a nivel mundial. Anualmente a nivel mundial se cultivan unos 20 millones de hectáreas de maní considerando los diferentes tipos (FAPRI, 2010).

El maní presenta crecimiento herbáceo indeterminado, su altura puede alcanzar entre $45-60 \mathrm{~cm}$ con 4 a 5 ramificaciones de importancia, sus hojas

\footnotetext{
1 Universidad de Concepción, Facultad de Agronomía, Av. Vicente Méndez 595, Casilla 537, Chillán, Chile.

* Autor para correspondencia: nzapata@udec
} 
son tetrafoliadas de tamaño y tonalidad variables con la variedad botánica y condiciones ambientales (Faiguenbaum, 1983). Las flores de esta especie están dispuestas en inflorescencias que se desarrollan en nudos foliares sobre las ramificaciones y/o tallo principal dependiendo de la variedad botánica (Leon, 2000). La floración abarca el $80 \%$ del ciclo evolutivo de la especie superponiéndose con la fructificación; una vez ocurrida la fecundación se produce la elongación del ovario fecundado hasta penetrar en el suelo donde se desarrollan los frutos (Leon, 2000). Debido a su hábito de crecimiento indeterminado, esta especie produce hojas continuamente, sustituyendo las que mueren en detrimento del crecimiento de los frutos o semillas (Fernández et al., 2006a). La acumulación de biomasa en la planta describe una curva sigmoidea típica que puede caer al final de la estación de crecimiento por condiciones ambientales menos favorables o por altas densidades (Giayetto et al., 2006).

El maní es un cultivo de establecimiento primaveral, no tolera heladas y su período vegetativo puede alcanzar los 160 a 180 días para genotipos cultivados en Chile (Faiguenbaum, 1983), sin embargo, también existen genotipos de ciclo corto que abarcan unos 130-150 días. La densidad de siembra es una de las prácticas de manejo que determina la capacidad del cultivo de interceptar recursos, pudiendo llegar a afectar de manera importante la captura y utilización de radiación, agua y nutrientes (Kruk y Satorre, 2003). La distribución espacial y la densidad de plantas no modifican el desarrollo fenológico de maní (Giayetto et al., 2003), aunque sí afectan la acumulación de materia seca por planta, independiente de la arquitectura propia de cada cultivar; densidades altas $\left(56 \mathrm{pl} \mathrm{m}^{-2}\right)$ derivan en una menor acumulación de biomasa aérea y de frutos por planta (Giayetto et al., 2006). Poblaciones de 25 a 30 plantas $\mathrm{m}^{-2}$ se indican como densidades óptimas para este cultivo, densidades mayores no mejoran el rendimiento de frutos ni los ingresos económicos (Giayetto et al., 2003). El cultivo de maní presenta un crecimiento inicial lento; una densidad de plantas adecuada implica que el maní ocupe rápidamente la totalidad del espacio disponible, logre un mejor crecimiento temprano y pueda competir exitosamente con las malezas (Fernández et al., 2006a). También se debe considerar que en condiciones ideales de cultivo un número de plantas inferior al óptimo afecta sensiblemente el rendimiento, por el contrario, el exceso de plantas no disminuye el rendimiento, pero aumenta el costo de establecimiento por concepto de semilla (Pedelini, 2008).

En Chile es posible establecer este cultivo exitosamente hasta los $37^{\circ}$ latitud sur (VIII Región), pudiendo transformarse en una alternativa productiva interesante para los agricultores de la zona centro y centro-sur de Chile. Sin embargo, este cultivo resulta desconocido para muchos agricultores ya que únicamente es desarrollado por pequeños agricultores de algunas zonas de VI Región (Faiguenbaum 1983). En Chile se cultiva principalmente maní tipo Español; sin embargo, no existe información actualizada para su establecimiento y manejo agronómico, lo que incluye la carencia de estudios sobre densidad poblacional y su impacto sobre el crecimiento y productividad de esta especie, lo que limita la entrega de recomendaciones técnicas acertadas a los productores para que mejoren sus rendimientos. Por lo antes indicado, este trabajo tuvo por objetivo estudiar el efecto de distintas densidades poblacionales sobre el crecimiento y productividad de maní tipo Español y tipo Virginia cultivados en la Provincia de Nuble, Chile.

\section{Materiales y Métodos}

Para este estudio se empleó una línea final de maní tipo Español y otra del tipo Virginia, ambas seleccionadas en la Facultad de Agronomía de la Universidad de Concepción. La semilla empleada proviene de cosechas realizadas el año anterior, con germinación del $95 \%$.

Los experimentos se realizaron la temporada 2009-2010 en la Estación Experimental "El Nogal" de la Universidad de Concepción, la cual se ubica en la Provincia de Nuble, VIII Región (36³5'56,87’'S, 72॰04'54,03" W y 140 m.s.n.m). El suelo del sitio experimental corresponde a la Serie Arrayán (medial, thermic Humic Haploxerands), con topografía plana y buen drenaje (Carrasco, 1998). El clima de esta localidad corresponde a mediterráneo temperado, con temperaturas mínimas y máximas anuales de 6,9 y $20,2{ }^{\circ} \mathrm{C}$, con un período libre de heladas de 5-6 meses y con una precipitación anual promedio de $1.200 \mathrm{~mm}$ (Del Pozo y Del Canto, 1999).

Con cada genotipo de maní se estableció un experimento, cada experimento comprendía cuatro tratamientos de densidad poblacional: 104, 139, 208 y 417 mil plantas ha $^{-1}$. Los experimentos fueron dispuestos en bloques completos al azar 
con cuatro repeticiones. La unidad experimental correspondía a una parcela de $12 \mathrm{~m}^{2}$ en sistema de hileras simples espaciadas a $60 \mathrm{~cm}$ (4 hileras de 5 $\mathrm{m}$ de largo). La siembra de los ensayos se realizó el 28 de octubre de 2009.

El suelo se preparó secuencialmente con arado de vertedera, rastra de discos y vibrocultivador, luego se abrieron surcos de siembra manualmente con binador hasta $8 \mathrm{~cm}$ de profundidad. En base a análisis químico de suelo del sitio experimental realizado antes de la preparación de suelo y considerando la demanda del cultivo, se aplicó una fertilización base en el fondo del surco equivalente por hectárea a $216 \mathrm{~kg}$ de fosfato diamónico (18-46-0), $166 \mathrm{~kg}$ de muriato de potasio (0-0-60) y $108 \mathrm{~kg}$ de cal Soprocal $\left(90 \% \mathrm{CaCO}_{3}\right)$. Una vez incorporado el fertilizante se distribuyeron las semillas, previamente desinfectadas con Captan ${ }^{\circledR} 80 \mathrm{WP}(500 \mathrm{~g} / 100 \mathrm{~kg}$ semilla), en los surcos a una profundidad de 3-4 $\mathrm{cm}$, según la densidad predeterminada considerando dos semillas por golpe; una vez ocurrida la emergencia las plántulas en exceso fueron eliminadas. La semilla no fue inoculada con bacterias fijadoras de nitrógeno; para suplir los requerimientos de este nutriente se aplicaron en estado de tres hojas 120 $\mathrm{kg} \mathrm{ha}^{-1}$ de urea $(46 \% \mathrm{~N})$ e igual dosis a inicios de floración. Durante todo el ciclo de crecimiento los experimentos se mantuvieron libres de malezas mediante control manual. A partir de diciembre y hasta fines de febrero se proporcionaron riegos homogéneos al cultivo con una frecuencia semanal. Para facilitar la formación de frutos se efectuaron dos aporcas durante el período de cultivo $\left(1^{\text {ra }}\right.$ semana de enero y $1^{\text {ra }}$ semana febrero). La cosecha de los experimentos se efectuó el 29 de marzo de 2010, es decir, 152 días después de siembra (DDS).

Durante el desarrollo del cultivo a los 73, 104, 118,131 y 152 DDS se extrajeron dos plantas en competencia perfecta desde cada parcela; para cada planta se determinó área foliar, biomasa seca total y biomasa seca de hojas, tallos, raíces y frutos. Para la última fecha muestreada también se determinó producción de semillas y número de hojas, tallos, frutos y semillas e índice de cosecha (IC). A cosecha se extraerán las plantas contenidas en $3 \mathrm{~m}$ lineales de las dos hileras centrales de cada parcela $(3,6$ $\mathrm{m}^{2}$ ); para la superficie cosechada se determinó peso seco de semillas, frutos y de 1.000 semillas, peso seco de parte aérea.

Los datos obtenidos en los experimentos fueron sometidos a análisis de varianza (ANDEVA) y sus medias fueron comparadas mediante el Test de Tukey $(p \leq 0,05)$. Los valores obtenidos en las evaluaciones realizadas a las dos plantas muestreadas fueron promediados y considerados como un solo dato para el análisis estadístico. Para el análisis de datos se empleó el programa estadístico Statgraphics ${ }^{\circledR}$ (STSC, 1987). Cuando fue necesario y para cumplir con las premisas del ANDEVA los datos fueron transformados por $\sqrt{ }(\mathrm{x}+0,5)$ (Little y Hills, 1976).

\section{Resultados y Discusión}

\section{Evolución del área foliar y materia seca acumulada por planta}

El incremento de la densidad poblacional en ambos genotipos de maní evaluados tuvo un efecto negativo sobre el crecimiento individual de cada planta en lo referente a biomasa acumulada, área foliar, número de tallos, hojas y estructuras reproductivas. A los 73 DDS el área foliar por planta fue similar para todas las densidades estudiadas en ambos genotipos; sin embargo, durante el desarrollo posterior del cultivo las plantas sometidas a las menores densidades alcanzaron mayor área foliar (Figura 1). El área foliar acumulada por planta para el genotipo Español se incrementó linealmente desde los 73 a 131 DDS, posteriormente el aumento fue más acotado. En el genotipo Virginia el área foliar presentó un incremento lineal hasta la cosecha (152 DDS). El área foliar acumulada a cosecha, en el tratamiento de menor densidad poblacional estudiada, fue de $5.729 \mathrm{~cm}^{2}$ planta $^{-1}$ para el genotipo Español y de $6.061 \mathrm{~cm}^{2}$ planta $^{-1}$ para el genotipo Virginia. A su vez, la acumulación de materia seca por planta, tanto para genotipo Español como Virginia, mostró un incremento similar entre los 73 a 131 DDS, posteriormente aumentó con mayor velocidad alcanzando a cosecha en el genotipo Español 108,10 g planta $^{-1}$ y en el genotipo Virginia $114,90 \mathrm{~g} \mathrm{planta}^{-1}$ (Figura 1). De modo coincidente con el área foliar, la acumulación de biomasa por planta disminuyó significativamente al aumentar la densidad poblacional. Este comportamiento obedece a que las plantas dentro de un diseño de cultivo deben competir por una cantidad limitada de recursos, por lo cual un mayor número de individuos presentes implica necesariamente mayores dificultades para su crecimiento y tal efecto puede acentuarse en la fase final de su desarrollo (Giayetto et al., 1998; Giayetto et al., 2006). 
Español

Virginia

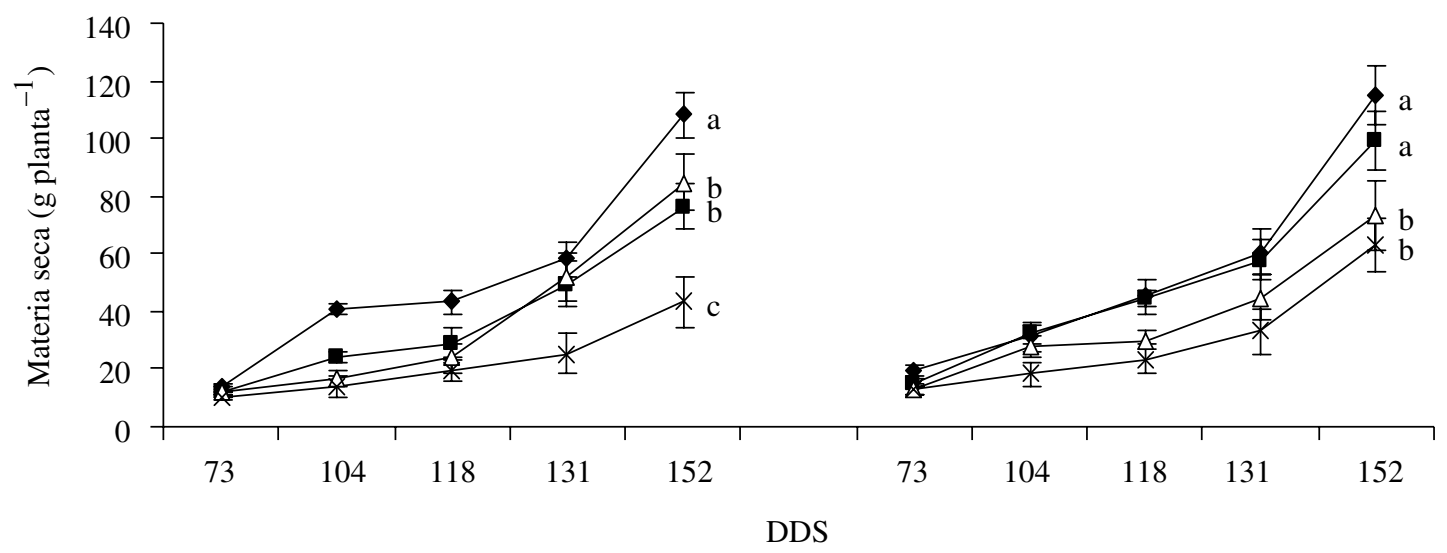

Español

Virginia

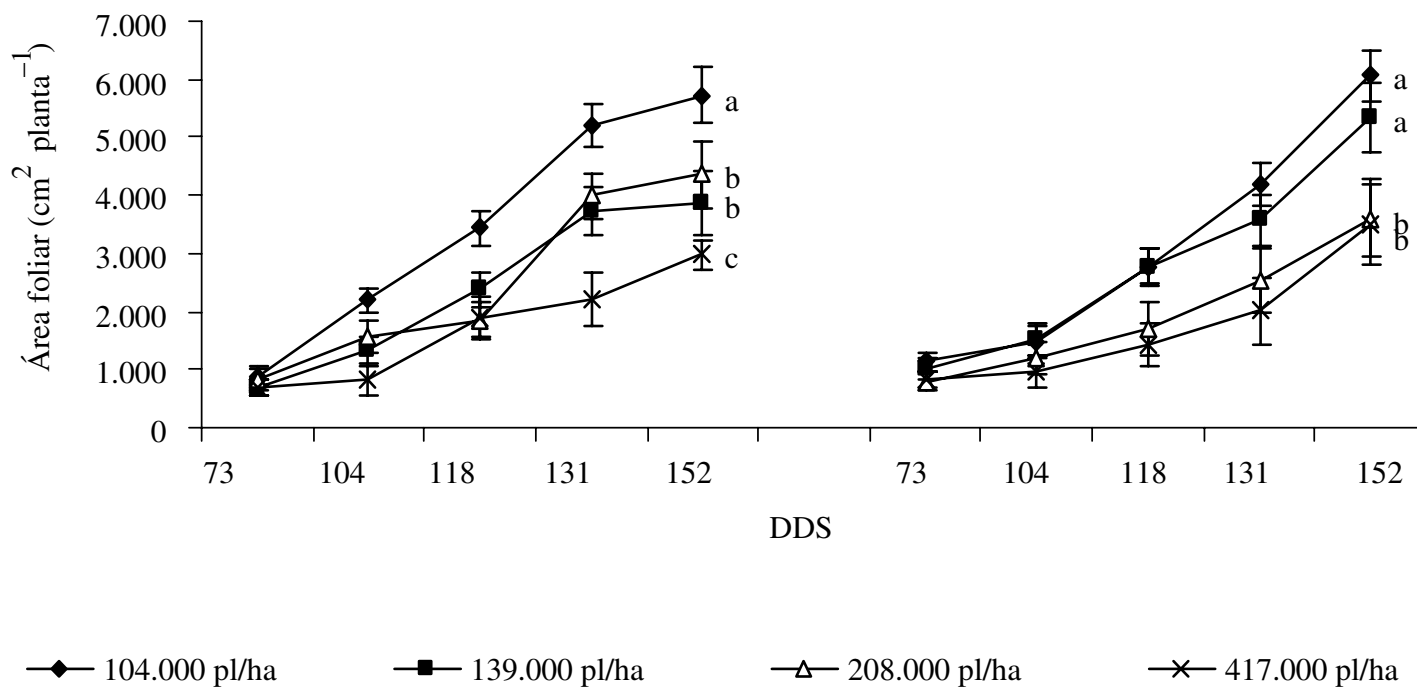

Figura 1. Materia seca acumulada y área foliar para dos genotipos de maní, según densidad poblacional, a distintos días después de siembra (DDS).

\section{Evolución de la distribución de biomasa por planta}

La distribución de biomasa en la planta no varió sustancialmente con el aumento de la densidad poblacional (Figura 2). Independientemente del genotipo y densidad poblacional en estudio, entre los 73 y 131 DDS las hojas representaron la mayor proporción de biomasa acumulada por planta, por el contrario, la menor proporción estuvo dada por las raíces. Según se avanzó en el desarrollo del cultivo, los frutos fueron incrementando su participación respecto al total de biomasa acumulada en la planta, a los 131 DDS los frutos sólo fueron superados por las hojas.

$\mathrm{Al}$ momento de la cosecha, es decir, transcurridos 152 DDS, los frutos representaron la mayor proporción de biomasa seca en la planta para ambos 

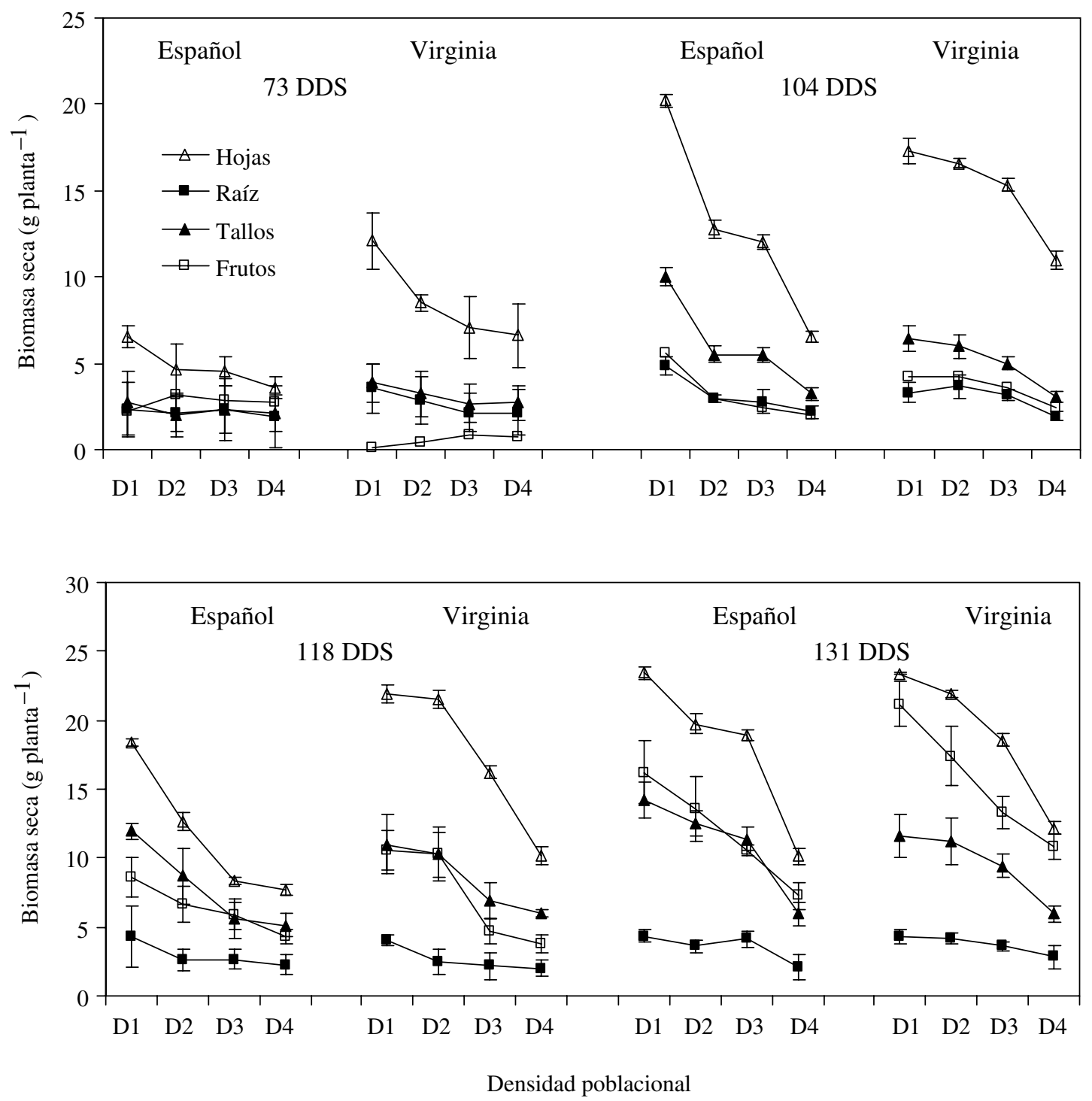

Figura 2. Distribución de biomasa en maní tipos Español y Virginia, según densidad poblacional, a distintos días después de siembra (DDS).

genotipos; hojas y tallos se ubicaron en segundo y tercer lugar de importancia (Tabla 1). La materia seca acumulada en los frutos representó entre el 37 y $45 \%$ para el genotipo Español, y entre el 35 y $44 \%$ para el genotipo Virginia, para la menor y mayor densidad poblacional respectivamente. Es decir, el incremento de la densidad implicó una mayor participación de los frutos en el total de materia seca acumulada por la planta, lo cual tuvo repercusión en incrementos significativos del índice de cosecha, los que variaron entre 0,24 y 0,31 para el genotipo Español y entre 0,26 y 0,37 para el genotipo Virginia (Tabla 1). Para este cultivo se indica que el índice de cosecha varía considerablemente dependiendo del cultivar; sin embargo, parece ser que altas densidades también favorecen su incremento en cultivares precoces, pudiendo ser este el caso (Bell et al., 1991).

De acuerdo con lo observado en el presente estudio la densidad poblacional no influye en la distribución de la biomasa en la planta de maní, este mismo comportamiento ha sido previamente 
Tabla 1. Partición de biomasa al momento de cosecha (152 DDS) de dos genotipos de maní cultivados bajo distintas densidades poblacionales.

\begin{tabular}{|c|c|c|c|c|c|c|}
\hline \multirow{2}{*}{$\begin{array}{l}\text { Población } \\
\left(\text { Plantas ha }^{-1}\right)\end{array}$} & \multicolumn{5}{|c|}{ Materia seca $\left(\mathrm{g}_{\text {planta }}{ }^{-1}\right)$} & \multirow{2}{*}{ IC } \\
\hline & Hojas & Tallos & Raíces & Frutos & Semillas & \\
\hline \multicolumn{7}{|c|}{ Genotipo Español } \\
\hline 104.000 & $27,83 \pm 1,35 \mathrm{a}$ & $19,36 \pm 1,80 \mathrm{a}$ & $4,63 \pm 0,61 \mathrm{a}$ & $31,00 \pm 1,91 \mathrm{a}$ & $20,45 \pm 1,45 \mathrm{a}$ & $0,24 \mathrm{~b}$ \\
\hline 139.000 & $16,06 \pm 1,12 b$ & $12,48 \pm 1,72 b$ & $2,26 \pm 0,40 b$ & $23,71 \pm 3,25 b$ & $14,61 \pm 1,81 \mathrm{a}$ & $0,26 \mathrm{~b}$ \\
\hline 208.000 & $12,38 \pm 2,05 b$ & $9,49 \pm 2,75 b c$ & $2,12 \pm 0,42 b$ & $17,52 \pm 2,58 b$ & $11,30 \pm 0,95 b$ & $0,27 \mathrm{~b}$ \\
\hline 417.000 & $8,28 \pm 1,54 \mathrm{c}$ & $6,62 \pm 1,14 \mathrm{c}$ & $2,04 \pm 0,35 b$ & $13,75 \pm 2,07 \mathrm{c}$ & $9,40 \pm 1,29 b$ & $0,31 \mathrm{a}$ \\
\hline \multicolumn{7}{|c|}{ Genotipo Virginia } \\
\hline 104.000 & $30,47 \pm 1,41 \mathrm{a}$ & $20,42 \pm 0,89 a$ & $6,21 \pm 0,33 \mathrm{a}$ & $30,78 \pm 3,76 \mathrm{a}$ & $22,85 \pm 2,41 \mathrm{a}$ & $0,26 \mathrm{~d}$ \\
\hline 139.000 & $22,08 \pm 2,02 b$ & $14,27 \pm 2,09 \mathrm{~b}$ & $4,02 \pm 0,22 b$ & $26,71 \pm 1,39 \mathrm{a}$ & $19,46 \pm 1,92 \mathrm{ab}$ & $0,29 \mathrm{c}$ \\
\hline 208.000 & $11,77 \pm 0,91 \mathrm{c}$ & $7,55 \pm 0,73 \mathrm{c}$ & $2,47 \pm 0,27 \mathrm{c}$ & $15,47 \pm 3,02 b$ & $12,31 \pm 1,56 \mathrm{ab}$ & $0,33 \mathrm{~b}$ \\
\hline 417.000 & $7,71 \pm 2,26 \mathrm{~d}$ & $5,97 \pm 1,48 \mathrm{c}$ & $2,17 \pm 0,53 \mathrm{c}$ & $12,58 \pm 4,84 b$ & $10,56 \pm 3,86 b$ & $0,37 \mathrm{a}$ \\
\hline
\end{tabular}

Letras distintas en la misma columna indican que los valores difieren significativamente. ANDEVA, test de Tukey $(\mathrm{P} \leq 0,05)$. DDS: Días después de siembra.

IC: Índice de cosecha.

informado para diferentes tipos comerciales de maní (Giayetto et al., 1998). Se considera más bien que la distribución de la biomasa en este cultivo está condicionada por factores ambientales como temperatura y disponibilidad hídrica, entre otros (Fernández et al., 2006b). Independientemente de la densidad, la distribución de la biomasa en la planta de maní mostró el mismo comportamiento observado en estudios previos (Cholaky et al., 1984; Cholaky et al., 1998; Fernández et al., 2006a). En la primera etapa de crecimiento y durante el período vegetativo, las hojas en expansión y raíces fueron el principal destino de los fotoasimilados, en cambio en la fase reproductiva las hojas y ramificaciones enviaron fotoasimilados principalmente hacia los frutos.

El número de hojas, tallos, frutos y semillas por planta, cuantificado a los 152 DDS, descendió considerablemente con el aumento de la densidad poblacional, independiente del genotipo evaluado (Figura 3). Con la menor densidad estudiada, el genotipo Español alcanzó 134 hojas por planta y el tipo Virginia 126 hojas, en cambio con la mayor densidad se contabilizaron 54 y 61 hoja planta ${ }^{-1}$ para estos genotipos respectivamente. Igual tendencia se observó con el número de tallos planta ${ }^{-1}$, los cuales bajaron de un promedio de 13 a 6 de mayor a menor densidad poblacional. Menores densidades poblacionales también incidieron en un mayor número de frutos y semillas por planta. El aumento del número de estructuras por planta a menores densidades poblacionales explicaría en parte la mayor capacidad de acumulación de biomasa por la planta (Giayetto et al., 1998), lo cual no se traduce necesariamente en mayor productividad por unidad de superficie.

\section{Rendimiento y producción de biomasa total por unidad de superficie}

El incremento de la densidad poblacional en ambos genotipos de maní influyó positivamente en el rendimiento y acumulación de biomasa total por unidad de superficie (Tabla 2). El rendimiento de frutos obtenido entre la menor y mayor densidad estudiada fluctuó entre 1.946 y $3.445 \mathrm{~kg} \mathrm{ha}^{-1}$ para el genotipo Español y entre 1.426 y $3.537 \mathrm{~kg} \mathrm{ha}^{-1}$ para el genotipo Virginia. El incremento de la densidad poblacional también repercutió en un mayor rendimiento de semilla, obteniéndose para la mayor densidad ensayada 2.031 y $1.932 \mathrm{~kg} \mathrm{ha}^{-1}$ para los genotipos Español y Virginia, respectivamente. Con el aumento de la densidad poblacional también se evidenció una mayor producción de follaje y raíces; de menor a mayor densidad, la productividad de estas estructuras fluctuó entre 4.773 y $8.365 \mathrm{~kg} \mathrm{ha}^{-1}$ para el genotipo Español y entre 5.387 y 10.029 $\mathrm{kg} \mathrm{ha}^{-1}$ para el genotipo Virginia. El incremento de la densidad poblacional, para ambos genotipos estudiados, no disminuyó significativamente el peso de 1.000 semillas. 

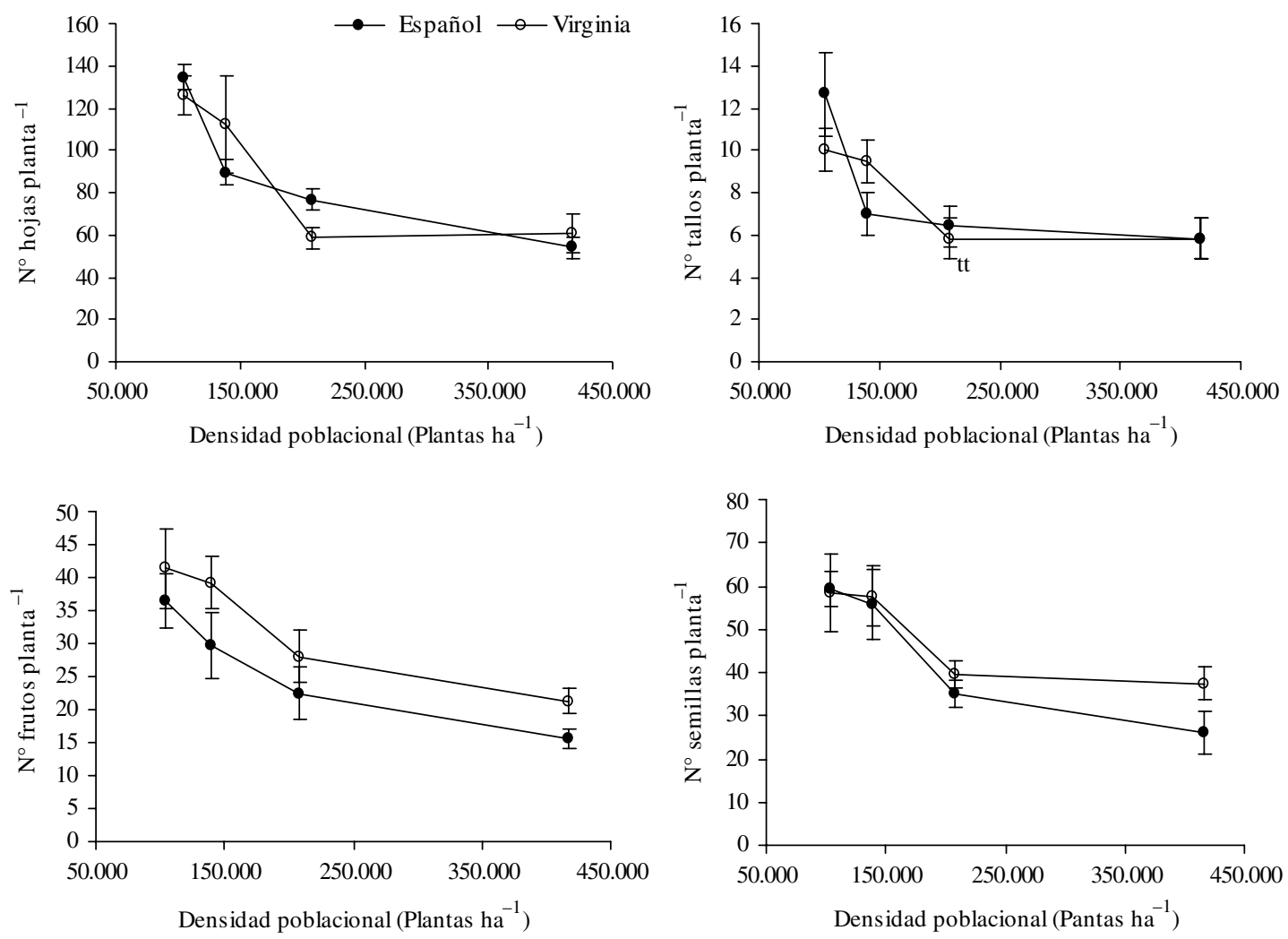

Figura 3. Número total de estructuras vegetativas y reproductivas por planta, según densidad poblacional, para dos genotipos de maní al momento de cosecha.

Tabla 2. Rendimiento de dos genotipos de maní establecidos bajo diferentes densidades poblacionales.

\begin{tabular}{lcccc}
\hline $\begin{array}{l}\text { Densidad } \\
\left(\text { Plantas } \mathrm{ha}^{-1}\right)\end{array}$ & Semilla & Frutos & Parte aérea y raíces & $\begin{array}{c}1.000 \text { semillas } \\
(\mathrm{g})\end{array}$ \\
\cline { 2 - 3 } & & & \\
& & & \\
& & & \\
$\left.1 \mathrm{~kg} \mathrm{ha}^{-1}\right)$ & & & \\
104.000 & $1.089 \pm 56 \mathrm{a}$ & $1.946 \pm 114 \mathrm{a}$ & $4.773 \pm 274 \mathrm{a}$ & $402 \pm 2 \mathrm{a}$ \\
139.000 & $1.240 \pm 100 \mathrm{a}$ & $2.179 \pm 160 \mathrm{a}$ & $5.581 \pm 511 \mathrm{ab}$ & $400 \pm 18 \mathrm{a}$ \\
208.000 & $1.496 \pm 73 \mathrm{~b}$ & $2.570 \pm 94 \mathrm{~b}$ & $6.432 \pm 279 \mathrm{~b}$ & $401 \pm 9 \mathrm{a}$ \\
417.000 & $2.031 \pm 55 \mathrm{c}$ & $3.445 \pm 96 \mathrm{c}$ & $8.365 \pm 219 \mathrm{c}$ & $391 \pm 16 \mathrm{a}$ \\
& & & \\
104.000 & $903 \pm 38 \mathrm{a}$ & $1.426 \pm 55 \mathrm{a}$ & $5.387 \pm 250 \mathrm{a}$ & $401 \pm 3 \mathrm{a}$ \\
139.000 & $978 \pm 105 \mathrm{a}$ & $1.642 \pm 227 \mathrm{ab}$ & $5.557 \pm 189 \mathrm{a}$ & $400 \pm 6 \mathrm{a}$ \\
208.000 & $1.443 \pm 144 \mathrm{~b}$ & $2.472 \pm 200 \mathrm{~b}$ & $7.311 \pm 624 \mathrm{~b}$ & $406 \pm 20 \mathrm{a}$ \\
417.000 & $1.932 \pm 288 \mathrm{c}$ & $3.537 \pm 333 \mathrm{c}$ & $10.029 \pm 462 \mathrm{c}$ & $397 \pm 13 \mathrm{a}$ \\
\hline
\end{tabular}

Letras distintas en la misma columna indican que los valores difieren significativamente. ANDEVA, test de Tukey $(\mathrm{P} \leq 0,05)$.

Los resultados obtenidos en el presente estudio indican que en igual superficie, las plantas desarrolladas a bajas densidades presentaron mayor productividad, pero ello no fue suficiente para equiparar la productividad de la superficie establecida con plantas en alta densidad. Esto posiblemente pueda explicarse por el hábito de crecimiento indeterminado que presentan estos genotipos, lo 
cual prolongó la fase reproductiva y aunque pudo haber gran cantidad de frutos en plantas establecidas a baja densidad, muchos frutos al momento de la cosecha estaban aún inmaduros, obteniéndose una baja eficiencia reproductiva por unidad de superficie (Fernández et al., 2006a).

$\mathrm{Si}$ se considera que, tanto para el genotipo Español como Virginia, la mayor densidad poblacional estudiada arrojó el mejor rendimiento de semilla y biomasa en general, esta densidad sería la más recomendable para establecimientos del cultivo bajo las condiciones ambientales estudiadas. El costo adicional en el que debería incurrir el agricultor por concepto de semilla para su establecimiento parece justificable, si se considera que podría obtener un rendimiento adicional de semilla de unos $500 \mathrm{~kg} \mathrm{ha}^{-1}$. El establecimiento de este cultivo en altas densidades también podría mejorar su competitividad frente a malezas. Sin embargo, este tema así como la respuesta al espaciamiento entre hileras para estos genotipos serán estudiados en próximas investigaciones.

\section{Conclusiones}

De la presente investigación se puede concluir que, tanto para el genotipo de maní Español como para Virginia, el aumento de la densidad poblacional disminuye significativamente el crecimiento y productividad por planta, expresados como área foliar, número de órganos y biomasa acumulada. En ambos genotipos de maní la distribución de biomasa en la planta no resultó afectada cuando se aumentó la densidad poblacional. Independiente del genotipo de maní evaluado, el incremento de la densidad poblacional aumentó significativamente la biomasa acumulada y rendimiento de semilla por unidad de superficie.

\section{Agradecimientos}

Los autores agradecen a la Dirección de Investigación de la Universidad de Concepción por financiar esta investigación. DIUC \# 211.122.022-1.0

\section{Literatura citada}

Bell, M.; Harch, G.; Wright, G

1991 Plant population studies on peanut (Arachis hypogaea L.) in subtropical Australia.1. Growth under fully irrigated conditions. Australian Journal of Experimental Agriculture 31: 535-543.

Carrasco, P.

1998 Descripciones de Suelos VIII Región. Chillán, Chile: Facultad de Agronomía Universidad de Concepción.

Cholaky, L.; Cantero, A.; Moreno, I.; Bonadeo, E.

1984 Acumulación de materia seca y absorción y distribución de N, P, K, Ca, Mg, Na y C en maní (Arachis hypogaea L.). Revista Universidad Nacional de Río Cuarto 4: 13-40.

Cholaky, L.; Giayetto, O.; Fernández, E.; Asnal E.

1998 Análisis de crecimiento de maní (Arachis hypogaea L.) tipos Valencia, Español y Virginia. Revista Universidad Nacional de Río Cuarto 18: 5-23.

Del Pozo, A.; Del Canto, P.

1999 Áreas agroclimáticas y sistemas productivos en la VII y VIII regiones. Chillán, Chile: INIA Quilamapu.

Faiguenbaum, $\mathrm{H}$.

1983 Cultivo del maní. Revista el Campesino 11422-29.

FAPRI

2010 U.S. and world agricultural Outlook. Iowa: Food and Agricultural Policy Research Institute Iowa State University, University of Missouri-Columbia, USA.

Fernández, E.; Giayetto, O.; Cholaky, L.

2006a Crecimiento y desarrollo. In Fernández, E., Giayetto, O. (eds.), El cultivo de maní en Córdoba. Universidad Nacional de Río Cuarto, Río Cuarto, Argentina, pp. 73-88.

Fernández, E.; Giayetto, O.; Cholaky, L.; Cerioni, G.

2006b Ecofisiología y factores ambientales, In: Fernández, E., Giayetto, O. (eds.), El cultivo de maní en Córdoba. Universidad Nacional de Río Cuarto. Río Cuarto, Argentina, pp. 89-112.
Giayetto, O.; Cerioni, G.; Asnal, W.

1998 Effect of Sowing Spacing on Vegetative Growth, Dry Matter Production, and Peanut Pod Yield. Peanut Science 25: 86-87.

Giayetto, O.; Ceroni, G.; Amín, M.

2003 Use of asymptotic model to obtain optimum plant density in peanut (Arachis hypogaea L.). Peanut Science and Technology 32: 5-13.

Giayetto, O.; Fernandez, E.; Cerioni, G.

2006 Fecha y modelos de siembra, In: Fernández, E., Giayetto, O. (eds.), El cultivo de maní en Córdoba. Universidad Nacional de Río Cuarto. Río Cuarto, Argentina, pp. 157-169.

Hauser, E.; Buchanan, G.

1981 Influence of row spacing, seedling rate and herbicide system on the competitiveness and yield of peanuts. Peanut Science 8: 74-81.

Knauft, D.; Norden, A.; Gorbet, D.

1987 Peanut. In Fehr, W. (ed.), Principles of cultivar development. Macmillan Publishing, NY, USA, pp. 346-384.

Kruk, B.; Satorre, E.

2003 Densidad y Arreglo Espacial del cultivo. In Satorre, E. et al. (eds.), Producción de Granos. Bases funcionales para su manejo. Editorial Facultad de Agronomía UBA, Buenos Aires, Argentina, pp. 279-318.

Leon, J.

2000 Botánica de los cultivos tropicales. IICA, San José, Costa Rica.

Little, T.; Hills, F

1976 Métodos estadísticos para la investigación en agricultura. Ed. Trillas. México, 270 p.

Pedelini, R.

2008 Maní: Guía práctica para su cultivo. Córdoba, Argentina: Instituto Nacional de Tecnología Agropecuaria, INTA Manfredi, Córdoba, Argentina. 